\title{
On a Three-Valued Logic with Blamey's Interjunction for the Formal Description of Atomic Transactions
}

\author{
Mateusz M. Radzki ${ }^{1}$
}

Received: 3 April 2019 / Accepted: 22 June 2019 / Published online: 9 July 2019

(c) The Author(s) 2019

\begin{abstract}
The article demonstrates that to describe the property of atomicity (one of the ACID property) of transactions in database systems, we need a three-valued logic with propositional connective characterized in the same way as Blamey's interjunction. However, the article explains that since Blamey's partial logic with interjunction is a logic without tautologies, it does not satisfy some salient conditions of being a logic of atomic transactions. The article introduces a logic of the considered kind, and provides an example of the formal exposition of the case of an atomic transaction. Finally, the article explains the philosophical significance of the introduced logic.
\end{abstract}

Keywords Atomicity · Atomic transactions · Blamey's partial logic · Interjunction · Three-valued logic

\section{Introduction}

All-or-nothing states are not something unusual. Consider, for example, a football match. Each team has eleven players. We can say that a team wins if and only if all of its players win, i.e., if and only if the first player wins and the second player wins, and the third player wins, and so on. Thus, a team fails if and only if it is not true that the first player wins and the second player wins, and the third player wins, and so on.

However, it might be argued that this situation cannot be described accurately by means of the classical conjunction. When we say that it is not true that the first player wins and the second player wins, and the third player wins, and so on, we mean that none of the players wins. It is clear that the case that, for example, two players of a particular team win and the rest of them fail, is excluded, and hence, the proposition that describes this case can be considered to be neither true nor false.

Mateusz M. Radzki

matradzki@wp.pl

1 Institute of Philosophy and Sociology, The Maria Grzegorzewska University, ul. Szczęśliwicka 40, 02-353 Warsaw, Poland 
Therefore, from such a point of view, the description of the win/defeat situations in the team-sports requires a non-classical logic.

In the article, we present an example of a three-valued propositional logic of atomic transactions which are executed, like a football match, in the all-or-nothing fashion. We demonstrate that to describe the property of atomicity (one of the ACID property) of transactions in database systems, we need a propositional connective that is characterized in the same way as Stephen Blamey's interjunction.

The ACID properties (short for atomicity, consistency preservation, isolation, and durability) allow applications developers to avoid failures of transaction executions. For example,

[...] in a banking context where debit/credit transactions are executed this means that no money is ever lost in electronic funds transfers and customers can rely on electronic receipts and balance statements. These cornerstones for building highly dependable information systems can be successfully applied outside the scope of online transaction processing and classical database applications as well (Vossen 2009, p. 19).

In this article, we consider the property of atomicity. In an atomic transaction, either all operations occur, or none of them occurs. Therefore, the database treats all atomic operations as a one whole unit. In other words, an atomic transaction appears to be indivisible. Thus:

From the perspective of a client and an application program, a transaction is executed completely or not at all, i.e., in an all-or-nothing fashion. So the effects of a program under execution on the underlying data server(s) will only become visible to the outside world or to other program executions if and when the transaction reaches its "commit" operation. [...] On the other hand, if the program is abnormally terminated before reaching its commit operation, the data in the underlying data servers will be left in or automatically brought back to the state in which it was before the transaction started, i.e., the data appears as if the transaction had never been invoked at all (Vossen 2009, p. 20; cf. Lynch et al. 1993).

Therefore, either the transaction is executed from the initial state to the final state, or the system appears as if it had never left the initial state (cf. Lynch et al. 1988, p. 41). This can be captured by means of temporal logic in the following way:

$$
\operatorname{atomic}(t) \text { iff }\left(\left((\operatorname{pret}(t)=\sigma) \wedge\left(\sigma \rightarrow_{t} \sigma^{\prime}\right)\right) \rightarrow\left((\operatorname{post}(t)=\sigma) \vee\left(\operatorname{post}(t)=\sigma^{\prime}\right)\right)\right) \text {, }
$$

where atomic $(t)$ stands for an atomic transaction, $\operatorname{pret}(t)$ and $\operatorname{post}(t)$ stand for the systems' state before (respectively, after) the execution of transaction $t$, and $\sigma \rightarrow_{t} \sigma^{\prime}$ is the specification of $t$ from the system's state $\sigma$ to the system's state $\sigma^{\prime}$ (Zarras and Issarny 1998, p. 261).

Although the above formula expresses that the final state of an atomic transaction either is equal to the initial state, or is equal to the "atomic commit", it does not capture the all-or-nothing feature of atomicity.

It is also worth noting that the well-known "transaction logic" (hereinafter TR) which provides a logical foundation for the phenomenon of states changes in logic 
programs and databases (cf. Bonner and Kifer 1995, 1998) by means of a new binary connective for sequential composition (called "serial conjunction", and symbolized by $\otimes$ ), does not provide a formal tool for describing the "atomic commit". Thus, although TR extends the syntax of first-order predicate logic with $\otimes$, still it does not allow us to express the all-or-nothing feature of atomic transactions.

Hence, we shall introduce a special propositional connective \& defined as follows.

Definition 1 Propositional connective \& provides true and false formulae as follows:

$$
\begin{aligned}
& v\left(A_{1} \& A_{2}\right)=1 \text { iff } v\left(A_{1}\right)=1 \text { and } v\left(A_{2}\right)=1, \\
& v\left(A_{1} \& A_{2}\right)=0 \text { iff } v\left(A_{1}\right)=0 \text { and } v\left(A_{2}\right)=0,
\end{aligned}
$$

where $A_{1}$ and $A_{2}$ are arbitrary (not necessary distinct) formulae, FOR is a set of formulae, $v: F O R \rightarrow\{1, *, 0\}, 1$ means "true", and 0 means "false". In other cases of the valuation of $A_{1}$ and $A_{2}$, the valuation of $A_{1} \& A_{2}$ is equal to $*$, i.e., a third truthvalue without specified meaning. Thus, $\&$ is defined as a propositional connective of a three-valued logic whose formulae assign the following truth-values: 1,0 and $*$.

Hence, for two arbitrary arguments $A_{1}$ and $A_{2}$, \& is characterized by the following truth-table (Table 1):

It is easy to recognize that $\&$ is defined in the same way as Blamey's interjunction (symbolized by $\times \times$ ) in his "partial logic" (hereinafter PrL) (Blamey 2002, p. 264). In PrL, the third truth-value means a "truth-value gap".

However, since the connectives of PrL, i.e., $\sim, \rightarrow, \vee, \wedge, \leftrightarrow$ are defined in the same way as Stephen C. Kleene's "strong” connectives (Blamey 2002, p. 267), PrL, like Kleene's three-valued "strong" logic, is a logic that contains no tautologies (Bolc and Borowik 1992, p. 74). Consequently, some relevant from a view of the presented reasoning formulae constructed out of $\times \times$ are not tautologies, for example,

$$
\sim(p \times \times q) \leftrightarrow(\sim p \times \times \sim q)
$$

Table 1 Propositional connective \&

\begin{tabular}{lll}
\hline$A_{1}$ & $A_{2}$ & $A_{1} \& A_{2}$ \\
\hline 0 & 0 & 0 \\
0 & $*$ & $*$ \\
0 & 1 & $*$ \\
$*$ & 0 & $*$ \\
$*$ & $*$ & $*$ \\
$*$ & 1 & $*$ \\
1 & 0 & $*$ \\
1 & $*$ & $*$ \\
\hline
\end{tabular}


As we shall see, for the sake of the formal exposition of atomic transactions, we will need a formula that corresponds to above formula as a tautology of our threevalued logic. Moreover, some other formulae constructed out of the familiar connectives will be required as tautologies as well, for example,

$$
((p \leftrightarrow q) \wedge(q \leftrightarrow r)) \rightarrow(p \leftrightarrow r) .
$$

Thus, an "atomic commit" cannot be described by means of PrL with $\times \times$. Hence, Sect. 2 presents an example of a three-valued logic that satisfies conditions for being a "logic of atomic transactions" (hereinafter AtL), Sect. 3 provides an example of the formal exposition of an atomic transaction, and Sect. 4 concerns the philosophical meaning of AtL. In the "Appendix", we shall present a proof of a theorem of AtL that is crucial in the presented reasoning.

\section{Logic of Atomic Transactions}

Now, let $\mathrm{L}_{\mathrm{AtL}}$ be a propositional language of AtL defined as the following algebra:

$$
\mathrm{L}_{\mathrm{AtL}}=\left(\mathrm{FOR}_{\mathrm{AtL}}, \sim, \rightarrow, \vee, \wedge, \leftrightarrow, \&\right),
$$

where $\mathrm{FOR}_{\mathrm{AtL}}$ is a set of wffs of $\mathrm{L}_{\mathrm{AtL}}$.

Let $\mathrm{M}_{\mathrm{AtL}}$ be a logical matrix for AtL, i.e.

$$
\mathrm{M}_{\mathrm{AtL}}=\left(\mathrm{A}_{\mathrm{AtL}},\{1\}\right)
$$

where $\mathrm{A}_{\mathrm{AtL}}=(\{1, *, 0\}, \sim, \rightarrow, \vee, \wedge, \leftrightarrow, \&)$. Let $p$ and $q$ be propositional variables of AtL. Then, let us characterize the propositional connectives $\sim, \rightarrow, \vee, \wedge, \leftrightarrow \&$ by the following truth-tables (Table 2):

Table 2 Propositional connectives of AtL

\begin{tabular}{lllllll}
\hline$p$ & & & & & $\sim p$ \\
\hline 0 & & & & & & 1 \\
$*$ & & & & & $*$ \\
1 & & & & & & 0 \\
\hline$p$ & $q$ & $p \vee q$ & $p \vee q$ & $p$ & $p$ & $p \& q$ \\
\hline 0 & 0 & 1 & 0 & 0 & 1 & 0 \\
0 & $*$ & 1 & 1 & 0 & 0 & $*$ \\
0 & 1 & 1 & 1 & 0 & 0 & $*$ \\
$*$ & 0 & $*$ & 1 & 0 & 0 & $*$ \\
$*$ & $*$ & 1 & 1 & 0 & 1 & $*$ \\
1 & 1 & 1 & 1 & 0 & 0 & $*$ \\
1 & 0 & 0 & 1 & 0 & 0 & $*$ \\
1 & 1 & 1 & 1 & 0 & 0 & 1 \\
\hline
\end{tabular}


The connectives $\sim$ and $\rightarrow$ are defined in the same way as the connectives $\sim$ and $\rightarrow$, respectively, of the three-valued logic of Jan Łukasiewicz (hereinafter $\mathrm{L}_{3}$ ) (Łukasiewicz 1967, p. 54). However, $\vee, \wedge, \leftrightarrow$ are defined in the more "classical" way-formulae which are constructed out of them do not take on the truth-value

Hence, the following formulae are examples of tautologies of AtL that correspond to some basic tautologies of the classical propositional logic (hereinafter CL):

$$
\begin{gathered}
p \rightarrow p, \\
p \leftrightarrow p, \\
p \rightarrow \sim \sim p, \\
\sim \sim p \rightarrow p, \\
p \leftrightarrow \sim \sim p, \\
\sim(p \wedge \sim p), \\
(p \wedge q) \rightarrow p, \\
(p \wedge \sim p) \rightarrow q, \\
((p \rightarrow q) \wedge p) \rightarrow q, \\
p \vee \sim p, \\
p \rightarrow(p \vee q), \\
(p \wedge q) \leftrightarrow \sim(\sim p \vee \sim q), \\
(p \vee q) \leftrightarrow \sim(\sim p \wedge \sim q), \\
(p \leftrightarrow q) \leftrightarrow((p \rightarrow q) \wedge(q \rightarrow p)), \\
(p \leftrightarrow q) \leftrightarrow(\sim p \leftrightarrow \sim q) .
\end{gathered}
$$

On the other hand, some other basic tautologies of CL do not correspond to tautologies of AtL. For example, the following formulae are not tautologies of AtL:

$$
\begin{gathered}
(p \rightarrow \sim p) \rightarrow \sim p, \\
((p \wedge q) \rightarrow r) \leftrightarrow((p \wedge \sim q) \rightarrow \sim r) .
\end{gathered}
$$

However, what is the most salient from a view of the presented exposition is that the following formulae are tautologies of AtL:

$$
\begin{gathered}
\sim(p \& q) \rightarrow(\sim p \& \sim q), \\
(\sim p \& \sim q) \rightarrow \sim(p \& q), \\
\sim(p \& q) \leftrightarrow(\sim p \& \sim q), \\
((p \leftrightarrow q) \wedge(p \& r)) \rightarrow(q \& r),
\end{gathered}
$$




$$
\begin{aligned}
& ((p \rightarrow q) \wedge(q \rightarrow p)) \rightarrow(p \leftrightarrow q) \\
& ((p \leftrightarrow q) \wedge(q \leftrightarrow r)) \rightarrow(p \leftrightarrow r) .
\end{aligned}
$$

It should be noted that in AtL, the following definitions hold:

\section{Definition 2}

$$
(p \vee q) \stackrel{\mathrm{DEF}}{\leftrightarrow} \sim(\sim p \wedge \sim q),
$$

\section{Definition 3}

$$
(p \wedge q) \stackrel{\mathrm{DEF}}{\leftrightarrow} \sim(\sim p \vee \sim q),
$$

\section{Definition 4}

$$
(p \leftrightarrow q) \stackrel{\mathrm{DEF}}{\leftrightarrow}((p \rightarrow q) \wedge(q \rightarrow p)) .
$$

Therefore, in AtL, $\vee, \wedge, \leftrightarrow$ are definable in terms of $\sim, \rightarrow$. Consequently, to introduce an axiom system for AtL, one can use the Wajsberg axioms for $\mathrm{L}_{3}$ (cf. Wajsberg 1967) with additional axioms for theorems of AtL constructed out of \&.

Now, we can establish some structural features which characterize AtL. For example, it is not difficult to verify that the connectives of AtL that correspond to basic connectives of CL, i.e., $\sim, \rightarrow, \vee, \wedge, \leftrightarrow$ are "normal". This means that if every argument of a truth-function corresponding to any of them is either 1 or 0 , then this truth-function assumes either 1 or 0 (Rescher 1968, p. 78). On the other hand, since the truth-function corresponding to \& assumes for arguments 1 and $0, \&$ is not a "normal" connective. Moreover, AtL is, like $\mathrm{L}_{3}$, a "uniform", however, it is not a "regular in the sense of Kleene" (cf. Rescher 1968, pp. 78-80; 1969, pp. 55-58). AtL is also a "strongly uniform" (trivially so, since AtL is a three-valued logic), but, unlike $\mathrm{L}_{3}$, AtL is not a "continuous" many-valued propositional logic (cf. Rescher 1968, pp. 81-82; 1969, pp. 59-60).

It is worth noting that since in AtL, $\sim$ and $\rightarrow$ are defined in the same way as, respectively, $\sim$ and $\rightarrow$ in $L_{3}$, and all remaining connectives of $L_{3}$ are definable in terms of $\sim$ and $\rightarrow$ (cf. Malinowski 2007, p. 36; Urquhart 2011, p. 252), all connectives, not only those basic ones, that are definable in $\mathrm{L}_{3}$, are definable in AtL as well. Since, according to "standard" conditions established by John B. Rosser and Atwell $\mathrm{R}$. Turquette, $\mathrm{L}_{3}$ is a "standard" logic that has "standard" connectives as primitive or definable (Rosser and Turquette 1952, pp. 25-26; cf. Malinowski 2006, p. 552; Malinowski 2007, p. 41; Radzki 2017b, pp. 27-29), AtL is a "standard" logic as well.

Notice that special unary "standard" connectives, i.e., $j_{1}, j_{*}, j_{0}$ can be introduced by the following definitions:

\section{Definition 5}

$$
j_{1} p \stackrel{\mathrm{DEF}}{\leftrightarrow} \sim(p \rightarrow \sim p),
$$

\section{Definition 6}

$$
j_{*} \stackrel{\mathrm{DEF}}{\leftrightarrow}(p \leftrightarrow \sim p),
$$




\section{Definition 7}

$$
j_{0} p \stackrel{\mathrm{DEF}}{\leftrightarrow} \sim(\sim p \rightarrow p) .
$$

Standard negation in AtL, i.e., $\Rightarrow$ can be defined by

\section{Definition 8}

$$
\Rightarrow p \stackrel{\mathrm{DEF}}{\leftrightarrow} \sim j_{1} p .
$$

It should be noted that in AtL, $\wedge$ satisfies "standard" conditions. Then, "standard" disjunction, i.e., $\mathbb{V}$ can be defined by

\section{Definition 9}

$$
(p \mathbb{V} q) \stackrel{\mathrm{DEF}}{\leftrightarrow} \sqsupset(\neg p \wedge \neg q),
$$

"standard" implication in AtL, i.e., $\Rightarrow-$ by

\section{Definition 10}

$$
(p \Rightarrow q) \stackrel{\mathrm{DEF}}{\leftrightarrow} \Rightarrow(p \wedge \neg q),
$$

and standard equivalence in AtL, i.e., $\Leftrightarrow-$ by

\section{Definition 11}

$$
(p \Leftrightarrow q) \stackrel{\mathrm{DEF}}{\leftrightarrow}((p \Rightarrow q) \wedge(q \Rightarrow p)) .
$$

It is also worth noting that AtL, in contrary, for example, to $\mathrm{L}_{3}$, is a functionally complete three-valued logic. Jerzy Słupecki demonstrated (Słupecki, 1967) that every propositional connective of a functionally complete three-valued logic is definable in terms of $\{\sim, \rightarrow, T\}$, where $\sim$ is negation of $\mathrm{L}_{3}, \rightarrow$ is implication of $\mathrm{L}_{3}$, and $T$ is a unary connective characterized by the following truth-table (in $\mathrm{L}_{3}, 1 / 2$ is a third truth-value) (Table 3):

Recall that in AtL, $\sim$ and $\rightarrow$ are defined in the same way as, respectively, $\sim$ and $\rightarrow$ in $\mathrm{L}_{3}$. The Słupecki operator $T$ can be introduced to AtL by the following definition (at this point, for each argument, $T p$ instead of $1 / 2$ assigns $*$ ):

\section{Definition 12}

$$
T p \stackrel{\text { DEF }}{\leftrightarrow}(p \& \sim p) .
$$

\begin{tabular}{lc}
\hline$p$ & $T p$ \\
\hline 0 & $1 / 2$ \\
$1 / 2$ & $1 / 2$ \\
1 & $1 / 2$ \\
\hline
\end{tabular}


As we shall see in the next section, in the formal exposition of atomic transactions by means of AtL, three rules of inference are involved: the rule of substitution (hereinafter SUB), the rule of detachment (hereinafter MP), and the rule of conjunction introduction (hereinafter CI). Although in the presented reasoning, we do not introduce an axiom system for AtL, it is not difficult to verify that using AtL, we are allowed to apply these rules.

Since AtL is a truth-functional logic, SUB preserves the validity in AtL, and thus, if $A_{1}(p)$ is a tautology of AtL, then $A_{1}\left(A_{2}\right)$ is a tautology of AtL as well, where $A_{1}\left(A_{2}\right)$ is the result of substituting $A_{2}$ uniformly for $p$ in $A_{1}$ (cf. Epstein 2011). Since $\mathrm{MP}$ is truth-preserving in $\mathrm{L}_{3}$, the fact that MP is truth-preserving in AtL requires no explanation (recall that $\rightarrow$ works in the same way both in $\mathrm{L}_{3}$ and AtL), and hence, we are allowed to infer a formula of the form $A_{2}$ from two formulae of the forms $A_{1}$ and $A_{1} \rightarrow A_{2}$. Finally, it can be verified immediately by the Table 2 that CI is truthpreserving in AtL, and hence, we can infer a formula of the form $A_{1} \wedge A_{2}$ from two formulae of the forms $A_{1}$ and $A_{2}$. Consequently, in AtL, both MP and CI preserve the validity.

\section{Formal Exposition of the Case of an Atomic Transaction}

Now, let us examine how the introduced AtL deals with the formal exposition of the case of an atomic transaction. Let $p, q_{1}, q_{2}, \ldots, q_{n}, r(n \in \mathbb{N})$ be propositional variables of AtL. Suppose $p$ is interpreted by a proposition stating that certain atomic transaction, call it $a$, is executed, and each of $q_{1}, q_{2}, \ldots, q_{n}$ is interpreted by a proposition stating that certain operations of $a$, call it $o_{1}, o_{2}, \ldots, o_{n}$, respectively, occur.

Suppose $a$ consists of two operations $o_{1}$ and $o_{2}$.

Then, it can be established that

(1) $p \leftrightarrow\left(q_{1} \& q_{2}\right)$.

However, if $a$ is deleted, then

(2) $\sim p \leftrightarrow \sim\left(q_{1} \& q_{2}\right)$.

The formula (2) expresses that $a$ is deleted iff the whole sequence of operations of $a$ is deleted. But

(3) $\sim\left(q_{1} \& q_{2}\right) \leftrightarrow\left(\sim q_{1} \& \sim q_{2}\right)$

is a tautology of AtL. Thus, since $((p \leftrightarrow q) \wedge(q \leftrightarrow r)) \rightarrow(p \leftrightarrow r)$ is a tautology of AtL, by (2), (3), SUB, CI and MP, we obtain

(4) $\sim p \leftrightarrow\left(\sim q_{1} \& \sim q_{2}\right)$. 
The formula (4) expresses that $a$ is deleted iff each operation of $a$ is deleted, i.e., $o_{1}$ is deleted and $o_{2}$ is deleted.

It is easy to verify that AtL also deals with the formal exposition of the case that $a$ consists of any finite number of operations $o_{1}, o_{2}, \ldots, o_{n}$.

Then, instead of (1), we have

(5) $p \leftrightarrow\left(q_{1} \& q_{2} \& \ldots \& q_{n}\right)$.

Instead of (2), we get

(6) $\sim p \leftrightarrow \sim\left(q_{1} \& q_{2} \& \ldots \& q_{n}\right)$.

Then, we need

(7) $\sim\left(q_{1} \& q_{2} \& \ldots \& q_{n}\right) \leftrightarrow\left(\sim q_{1} \& \sim q_{2} \& \ldots \& \sim q_{n}\right)$.

The above formula can be obtained by the mathematical induction (see the formal details in the "Appendix").

Hence, instead of (4), we get

(8) $\sim p \leftrightarrow\left(\sim q_{1} \& \sim q_{2} \& \ldots \& \sim q_{n}\right)$.

In the presented exposition, the formula (3) (or the formula (7)) directly captures the mechanism of atomic transactions. It expresses that the whole sequence of any operations is deleted iff each operation is deleted.

Hence, in virtue of (3) (or (7)), from the formula expressing that the whole sequence of operations of $a$ is deleted, we can obtain the formula expressing that each operation of $a$ is deleted. In the same way, from the formula expressing that each operation of $a$ is deleted, we can obtain the formula expressing that the whole sequence of operations of $a$ is deleted. Consequently, the introduced AtL perfectly deals with the formal exposition of the case of an atomic transaction.

\section{Philosophical Significance of AtL}

Usually, the philosophical significance of an arbitrary many-valued propositional logic is condensed in the interpretation of additional (i.e., different from 1 which symbolizes the "true", and different from 0 which symbolizes the "false") truthvalues, or, as some logicians prefer to say, intermediate truth-degrees (cf. Gottwald 2001, p. 4).

Since the truth-degrees are introduced with certain justification (however, with some exceptions, for example, in many-valued logic of Emil Post (cf. Malinowski 2006, pp. 547-548)), usually they are the bearers of philosophical meaning. In other words, they encapsulate certain philosophical motivations for the investigations of many-valued systems. 
For example, in $\mathrm{L}_{3}$, the third truth-value $1 / 2$ refers to the contingent nature of the future states of affairs, and is assigned to propositions that are neither true nor false, but which are only possibly true and possibly false (i.e., contingently true; cf. Gottwald 1999, p. 12; Haack 1996, pp. 73-74; Łukasiewicz 1967, p. 53; Malinowski 2009, pp. 82-83; Mattila 2009, p. 272; Tooley 1997, p. 133). In the three-valued logic of Dmitrii A. Bochvar, the intermediate truth-valued $I$ is assigned to "paradoxical" or even "meaningless" propositions (cf. Malinowski 2007, p. 24; Rescher 1968, p. 67; Urquhart 2011, pp. 252-253). Bochvar's logical system was constructed as a formal tool for overcoming problems that follows from logical antinomies (Bolc and Borowik 1992, p. 65). The same idea is a founding one of the three-valued calculus of Victor K. Finn (Bolc and Borowik 1992, p. 66). In the logical system of Kleene, the third truth-valued is ascribed to "undetermined", "not defined" statements (cf. Bergmann 2008, p. 74; Malinowski 2007, pp. 22-23; Rescher 1968, p. 72; Urquhart 2011, pp. 253-254).

Let us take a closer look at Kleene's interpretation of the third truth-value. As Nicholas J. J. Smith explains:

Kleene was motivated by a consideration of partial recursive predicates. Think of such a predicate as coming with an algorithm. Given some objects as input, the algorithm terminates with the answer 'Yes': the predicate is true of these objects. Given some (other) objects as input, the algorithm terminates with the answer 'No': the predicate is false of these objects. But for some (other) objects, the algorithm does not terminate with either answer: the predicate is undefined for these objects. Hence Kleene three truth values: $\mathbf{t}$ (true), $\mathbf{f}$ (false) and $\mathbf{u}$ (undefined). [...] Kleene [...] also considers a different interpretation of his three values: " $\mathbf{t}, \mathbf{f}, \mathbf{u}$ must be susceptible of another meaning besides (i) 'true', 'false', 'undefined', namely (ii) 'true', 'false', 'unknown (or value immaterial)'. Here 'unknown' is a category into which we can regard any proposition as falling, whose valued we either do not know or choose for the moment to disregard; and it does not then the other two possibilities 'true' and 'false' (Smith 2012, pp. 653-654).

In the introduced AtL, the meaning of $*$ is close to the "truth-value gap" of Blamey's PrL, or to the mentioned above "unknown" of Kleene's system. Propositions that assign $\star$ are neither true nor false. Thus, we are released from the requirement of providing any specified interpretation of propositions that assign $\star$. Consequently, we can treat them as simply "disregarded".

It is also should be noted that the introduced AtL is more "classical" than, for example, the well-known $\mathrm{L}_{3}$. Many classical tautologies like the "rule of the excluded middle", the "rule of contradiction" and "modus ponens" which are not tautologies of $\mathrm{L}_{3}$, are tautologies of AtL. Consequently, AtL is not only an accurate calculus for the purpose that has been presented, but also it is a logical system that incorporates some fundamental intuitions concerning the classical "laws of logic" which are often considered to be the "laws of truth" (Frege 1956, p. 289). This feature may extend the application of AtL to all areas that in the formal exposition, require on one hand, a three-valued logic with $\&$, and, on the other hand, some basic "classical" laws of logic. 
Thus, we can consider AtL to be philosophically connected with CL. Such a property is not commonly shared by many-valued logics. It needs to be noted that many-valued logical systems, unlike, for example, modal logics, are usually treated as radically different from the classical logic, even from all classical modes of reasoning, not necessary logical ones. For example, Łukasiewicz compared his threevalued calculus to non-Euclidean geometry:

If $[\ldots]$ third valued is introduced into logic we change its very foundations. A trivalent system of logic, whose first outline I was able to give in 1920, differs from ordinary bivalent logic, the only one known so far, as much as nonEuclidean systems of geometry differ from Euclidean geometry (Łukasiewicz 1967, p. 37; cf. Łukasiewicz 1967, p. 63; Priest 2003, p. 442; Radzki 2017a, pp. 404-405; Urquhart 2011, p. 249).

Using the distinction introduced by Susan Haack, we can say that Łukasiewicz considered his three-valued logic as a "rival", not as a "supplement" to the classical logic (Haack 1996, pp. 2-3). As the other "rivals" we can indicate systems of Blamey, Bochvar, Finn, Heyting or Kleene. On the other hand, commonly investigated modal, epistemic, tense and deontic systems are usually treated as "supplements" to the classical logic. Although they contain new logical operators, for example, modal operators for necessity and possibility, they do not change the definitions of the classical propositional connectives, and thus, they leave the classical framework intact.

Although the introduced AtL is a three-valued logic, resemblance at the metalogical level between AtL and CL is quite deep. Therefore, AtL is not only an accurate calculus for the purpose that has been presented, but also it is a logical system philosophically consistent with some fundamental intuitions concerning the classical "laws of logic". Finally, AtL can be treated also as a "supplement" to some already developed transactional logics, for example, TR with connective for sequential composition.

\section{Conclusion}

To summarize, the introduced AtL is a three-valued propositional logic which is capable of describing all-or-nothing states-atomic transactions that are executed in the all-or-nothing fashion.

In the presented reasoning, the connective \& characterized in the same way as Blamey's interjunction is necessary to describe an "atomic commit". However, adding \& $($ or $X X)$ to PrL is not enough to construct AtL. We have demonstrated that some formulae are required to be tautologies of AtL. The most relevant is

$$
\sim\left(q_{1} \& q_{2} \& \ldots \& q_{n}\right) \leftrightarrow\left(\sim q_{1} \& \sim q_{2} \& \ldots \& \sim q_{n}\right)
$$

which captures the essence of atomic transactions, and enables us to present any of them as a one whole unit. 
The formal exposition of the case of atomic transaction by means of AtL is complementary to the formal exposition of such a case by means of temporal logic. The first one captures an all-or-nothing fashion of atomic transactions, on the other hand, the second one describes that the final state of an atomic transaction either is equal to the initial state, or is equal to the "atomic commit".

Finally, the introduced AtL is an example of an all-or-nothing logic that contains some fundamental classical "laws of logic", and hence, remains philosophically connected with CL. It is worth adding that this feature of AtL may extend the scope of its application to the formal expositions of other all-or-nothing states.

Open Access This article is distributed under the terms of the Creative Commons Attribution 4.0 International License (http://creativecommons.org/licenses/by/4.0/), which permits unrestricted use, distribution, and reproduction in any medium, provided you give appropriate credit to the original author(s) and the source, provide a link to the Creative Commons license, and indicate if changes were made.

\section{Appendix}

In this appendix, we shall demonstrate how to prove the formula (7) by means of the mathematical induction, some tautologies of AtL, SUB, CI and MP.

Proof Firstly, we need to obtain the following formula:

(a) $\sim\left(\left(q_{1} \& q_{2} \& \ldots\right) \& q_{n}\right) \leftrightarrow\left(\left(\sim q_{1} \& \sim q_{2} \& \ldots\right) \& \sim q_{n}\right)$.

Now, let us make the hypothesis of induction:

$$
\text { (ind.) } \sim\left(\left(q_{1} \& q_{2} \& \ldots\right) \& q_{k}\right) \leftrightarrow\left(\left(\sim q_{1} \& \sim q_{2} \& \ldots\right) \& \sim q_{k}\right) .
$$

Then, let us prove that

(b) $\sim\left(\left(q_{1} \& q_{2} \& \ldots\right) \& q_{n}\right) \rightarrow\left(\left(\sim q_{1} \& \sim q_{2} \& \ldots\right) \& \sim q_{n}\right)$.

Now, for $n=2$. It is not difficult to verify that $\sim\left(\left(q_{1}\right) \& q_{2}\right) \rightarrow\left(\left(\sim q_{1}\right) \& \sim q_{2}\right)$ is a tautology of AtL.

Then, suppose

(c) $\sim\left(\left(\left(q_{1} \& q_{2} \& \ldots\right) \& q_{k}\right) \& q_{k+1}\right)$.

Since $\sim(p \& q) \rightarrow(\sim p \& \sim q)$ is a tautology of AtL, by (c), SUB and MP, it follows that

(d) $\sim\left(\left(q_{1} \& q_{2} \& \ldots\right) \& q_{k}\right) \& \sim q_{k+1}$. 
Then, since $((p \leftrightarrow q) \wedge(p \& r)) \rightarrow(q \& r)$ is a tautology of AtL, by (ind.), (d), SUB, CI and MP, we obtain

(e) $\left(\left(\sim q_{1} \& \sim q_{2} \& \ldots\right) \& \sim q_{k}\right) \& \sim q_{k+1}$.

By $((p \& q) \& r) \rightarrow(p \& q \& r),(\mathrm{e}), \mathrm{SUB}$ and MP, it is inferred

(f) $\left(\sim q_{1} \& \sim q_{2} \& \ldots\right) \& \sim q_{k} \& \sim q_{k+1}$.

Therefore, we obtain $(\mathrm{c}) \rightarrow(\mathrm{f})$, and (b) is proved.

Now, let us verify that

(g) $\left(\left(\sim q_{1} \& \sim q_{2} \& \ldots\right) \& \sim q_{n}\right) \rightarrow \sim\left(\left(q_{1} \& q_{2} \& \ldots\right) \& q_{n}\right)$.

Now, for $n=2$. It is easy to find out that $\left(\left(\sim q_{1}\right) \& \sim q_{2}\right) \rightarrow \sim\left(\left(q_{1}\right) \& q_{2}\right)$ is a tautology of AtL.

Then, suppose

(h) $\left(\left(\left(\sim q_{1} \& \sim q_{2} \& \ldots\right) \& \sim q_{k}\right) \& \sim q_{k+1}\right)$.

Since $(p \& \sim q) \rightarrow \sim(\sim p \& q)$ is a tautology of AtL, by (h), SUB and MP, we have

(i) $\sim\left(\sim\left(\left(\sim q_{1} \& \sim q_{2} \& \ldots\right) \& \sim q_{k}\right) \& q_{k+1}\right)$.

Then, since $(\sim(\sim p \& q) \wedge(\sim r \leftrightarrow p)) \rightarrow \sim(r \& q)$ is a tautology of AtL, by (i), (ind.), SUB, CI and MP, it is inferred

(j) $\sim\left(\left(\left(q_{1} \& q_{2} \& \ldots\right) \& q_{k}\right) \& q_{k+1}\right)$.

By $\sim((p \& q) \& r) \rightarrow \sim(p \& q \& r),(\mathrm{j}), \mathrm{SUB}$ and MP, it follows that

(k) $\sim\left(\left(q_{1} \& q_{2} \& \ldots\right) \& q_{k} \& q_{k+1}\right)$.

Therefore, we obtain $(\mathrm{h}) \rightarrow(\mathrm{k})$, and $(\mathrm{g})$ is verified. Finally, by $((p \rightarrow q) \wedge(q \rightarrow p)) \rightarrow(p \leftrightarrow q),(\mathrm{b}),(\mathrm{g}), \mathrm{SUB}, \mathrm{CI}$ and MP, (a) is proved. 
The reader is asked to verify for himself that by means of the following tautologies of AtL:

$$
\begin{gathered}
\sim((p) \& q) \leftrightarrow \sim(p \& q), \\
((p \leftrightarrow q) \wedge(p \& r)) \rightarrow(q \& r), \\
((p \rightarrow q) \wedge(q \rightarrow p)) \rightarrow(p \leftrightarrow q),
\end{gathered}
$$

it is proved

(1) $\sim\left(\left(q_{1} \& q_{2} \& \ldots\right) \& q_{n}\right) \leftrightarrow \sim\left(q_{1} \& q_{2} \& \ldots \& q_{n}\right)$.

In the same way, by the following tautologies of AtL:

$$
\begin{gathered}
((p) \& q) \leftrightarrow(p \& q), \\
((p \leftrightarrow q) \wedge(p \& r)) \rightarrow(q \& r), \\
((p \rightarrow q) \wedge(q \rightarrow p)) \rightarrow(p \leftrightarrow q),
\end{gathered}
$$

we obtain

(m) $\left(\left(\sim q_{1} \& \sim q_{2} \& \ldots\right) \& \sim q_{n}\right) \leftrightarrow\left(\sim q_{1} \& \sim q_{2} \& \ldots \& \sim q_{n}\right)$.

Finally, since $((p \leftrightarrow q) \wedge(p \leftrightarrow r) \wedge(q \leftrightarrow s)) \rightarrow(r \leftrightarrow s)$ is a tautology of AtL, by (a), (1), (m), SUB, CI and MP, (7) is proved.

\section{References}

Bergmann M (2008) An introduction to many-valued and fuzzy logic. Cambridge University Press, Cambridge

Blamey S (2002) Partial logic. In: Gabbay M, Guenthner F (eds) Handbook of philosophical logic, vol 5, 2nd edn. Kluwer Academic Publishers, Dordrecht, pp 261-353

Bolc L, Borowik P (1992) Many-valued logics 1. Theoretical foundations. Springer, Berlin

Bonner AJ, Kifer M (1995) Transaction logic programming (or, a logic of procedural and declarative knowledge). University of Toronto: http://www3.cs.stonybrook.edu/ kifer/TechReports/transactio n-logic.pdf

Bonner AJ, Kifer M (1998) A logic for programming database transactions. In: Chomicki J, Saake G (eds) Logic for databases and information systems. Springer, New York, pp 117-166

Epstein RL (2011) Classical mathematical logic. The semantic foundations of logic. Princeton University Press, Princeton

Frege G (1956) The thought: a logical inquire. Mind N Ser 65(259):289-311

Gottwald S (1999) Many-valued logic and fuzzy set theory. In: Höhle U, Rodabaugh SE (eds) Mathematics of fuzzy sets. Logic, topology, and measure theory. Springer, New York, pp 5-90

Gottwald S (2001) A treatise on many-valued logics. Research Studio Press, Taunton 
Haack S (1996) Deviant logic, fuzzy logic. Beyond the formalism. The University of Chicago Press, Chicago

Łukasiewicz J (1967) Philosophical remarks on the many-valued systems of propositional logic. In: McCall S (ed) Polish logic 1920-1939. Clarendon Press, Oxford, pp 51-77

Lynch N, Merritt M, Weihl W, Fekete A (1988) A theory of atomic transactions. In: Gyssens M, Paredaens J, Van Gucht D (eds) 2nd International conference on database theory, Bruges, Belgium, August 31-September 2, 1988, Proceedings, pp 41-71

Lynch N, Merritt M, Weihl W, Fekete A (1993) Atomic transactions. In concurrent and distributed systems. Morgan Kaufmann, Burlington

Malinowski G (2006) Many-valued Logic. In: Jacquette D (ed) A companion to philosophical logic. Blackwell Publishing, Oxford, pp 545-561

Malinowski G (2007) Many-valued logic and its philosophy. In: Gabbay DM, Woods J (eds) Handbook of the history of logic, vol 8. The many valued and nonmonotonic turn in logic. North-Holland, Amsterdam, pp 13-94

Malinowski G (2009) A philosophy of many-valued logic. The third logical value and beyond. In: Lapointe $\mathrm{S}$ et al (eds) The golden age of polish philosophy. Logic, epistemology and the unity of science 16. Springer, New York, pp 81-92

Mattila JK (2009) Many-valuation, modality, and fuzziness. In: Seising R (ed) Views on fuzzy sets and systems. Springer, Berlin, pp 271-300

Priest G (2003) On alternative geometries, arithmetics, and logics; a tribute to Łukasiewicz. Stud Logica 74:441-468

Radzki MM (2017a) On axiom systems of Słupecki for the functionally complete three-valued logic. Axiomathes 27(5):403-415

Radzki MM (2017b) On the Rosser-Turquette method of constructing axiom systems for finitely manyvalued propositional logics of Łukasiewicz. J Appl Non Class Logics 27(4):27-32

Rescher N (1968) Topics in philosophical logic, Synthese library 17. Springer, Dordrecht

Rescher N (1969) Many-valued logic. McGraw-Hill, New York

Rosser JB, Turquette AR (1952) Many-valued logics. North-Holland, Amsterdam

Słupecki J (1967) The full three-valued propositional calculus. In: McCall S (ed) Polish logic 1920-1939. Clarendon Press, Oxford, pp 335-337

Smith JJ (2012) Many-Valued Logics. In: Russell G, Fara DG (eds) The Routledge companion philosophy of language. Routledge, New York, pp 636-651

Tooley M (1997) Time, tense, and causation. Clarendon Press, Oxford

Urquhart A (2011) Basic many-valued logic. In: Gabbay DM, Guenthner F (eds) Handbook of philosophical logic, vol 2, 2nd edn. Kluwer Academic Publishers, Dordrecht, pp 249-295

Vossen G (2009) ACID properties. In: Liu L, Özsu MT (eds) Encyclopedia of database systems. Springer, New York, pp 19-21

Wajsberg M (1967) Axiomatization of the three-valued propositional calculus. In: McCall S (ed) Polish logic 1920-1939. Clarendon Press, Oxford, pp 264-284

Zarras A, Issarny V (1998) A framework for systematic synthesis of transactional middleware. In: Davies N, Raymond K, Seitz J (eds) Middleware'98, IFIP international conference on distributed systems platforms and open distributed processing. Springer, Berlin, pp 257-272

Publisher's Note Springer Nature remains neutral with regard to jurisdictional claims in published maps and institutional affiliations. 